\title{
Research on the Topography Model of Micro Wire Electrical Discharge Machining Surface
}

\author{
Haijuan Ding ${ }^{1, a}$, Xiaohai $\mathrm{Li}^{1, \mathrm{~b}}$, Xinrong Wang ${ }^{1, \mathrm{c}}$, Libin Guo ${ }^{2, \mathrm{~d}}$, Liping Zhao ${ }^{1, \mathrm{e}}$ \\ ${ }^{1}$ College of Mechanical Engineering, Jiamusi University, Jiamusi, 154007, China \\ ${ }^{2}$ College of Mechanical Engineering, Harbin Engineering University, Harbin, 150001 China \\ adhj1000@126.com, 'lixh519@126.com, ' guolibin@hrbeu.edu.cn
} Keywords: Micro-WEDM; Surface Micro-topography; Mathematical Model; Discharge Crater;
Overlap Coefficient

\begin{abstract}
Micro Wire Electrical Discharge Machining (Micro-WEDM) surface consists of many approximate spherical peaks and craters with interphase distribution. According to the microWEDM mechanism, the surface micro-topography is simulated by using ANSYS, and the mathematical models about single crater and multi-crater are established. The formula of surface arithmetic average deviation $\mathrm{Sa}$ is deduced based on the overlap principle. It proves the validity of the surface micro-topography mathematical model comparing the simulation result with measurement calculation value from SPM, which can provide a new method and theoretical basis for evaluating the isotropy surface topography.
\end{abstract}

\section{Introduction}

Micro-WEDM is a kind of fine and high efficient machining method which has the characteristic of high cost-effective, untouched machining and high capability in machining three-dimensional micro-structure[1,2]. It plays a significant role in the micro electro-mechanical system manufacturing field such as microminiature spacecraft, microelectronic device and microminiature die.

The surface micro-topography can objectively reflect the fabrication mechanism and it has become the core content for researching surface quality. The micro-WEMD surface is formed by the wire electrode with random discharge. There is great difference on the formation mechanism with conventional machining methods. So the micro-topography of micro-WEDM surface has its own inherent characteristics. However, the surface structure and characteristics research has not established a whole assessment system. In this paper, the mathematical models for the micro-topography of micro-WEDM surface have been established to explore its geometric structure, and it will lay the foundation for the study on the micro-WEDM surface functional properties.

\section{Sample Preparation}

The material of sample is $\mathrm{W} 18 \mathrm{Cr} 4 \mathrm{~V}$ with the size of $1 \mathrm{~mm} \times 1 \mathrm{~mm} \times 1.5 \mathrm{~mm}$. The micro-WEDM tool (HIT100) is made by Harbin Institute of Technology using RC pulse power supply and oil-based working liquid. The wire electrode is tungsten wire with the diameter of $30 \mu \mathrm{m}$.

\section{Mathematical Model of Single Crater}

The discharge crater is similar to the shape of sphere in single pulse because of melting and exploding in micro-WEDM [3]. The melt material piles up around the crater and forms the lug boss. Fig. 1 shows the mathematical model of single crater, where the diameter of crater is $D$ and the depth is $H$.

The simulation result about crater depth and diameter is obtained by using ANSYS[4-6]. The discharge energy is larger, the depth is deeper and the diameter is greater and much narrower tending to ellipse, as shown in Fig.2. Here, $D_{1}$ is the diameter along the direction of wire electrode 
movement and $D_{2}$ is along the cross feed.

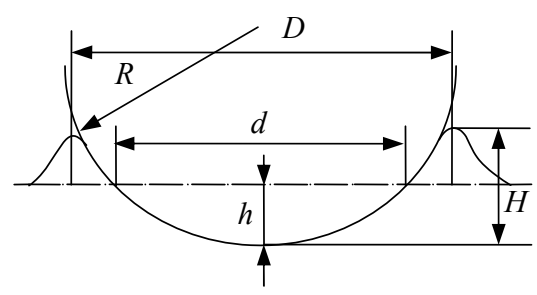

Fig.1 The mathematical model of the single pulse crater

Fig. 3 shows the variation trend of discharge crater size with different energy. With the increase of discharge energy, the diameter and depth of single pulse crater increase separately. And the increasing speed is slower when the energy reaches $10 \mu \mathrm{J}$. The major-minor axis ratio of crater $k_{1}$ and the ratio of depth-diameter $k_{\mathrm{h}}$ have the same increasing trend with increasing energy, and the variation range of $k_{1}$ is about $1 \sim 1.2$, as shown in Fig. 4 .

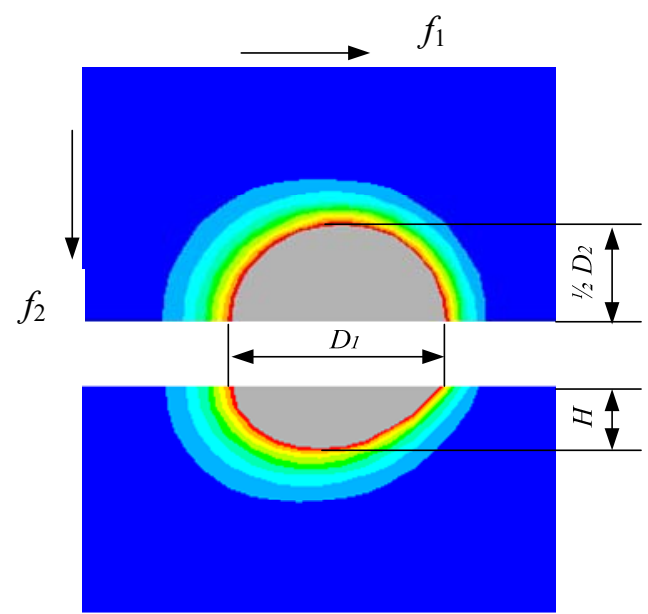

(a) $6.05 \mu \mathrm{J}, H=2.7 \mu \mathrm{m}$ $k_{1}=1.116, k_{\mathrm{h}}=0.6667$

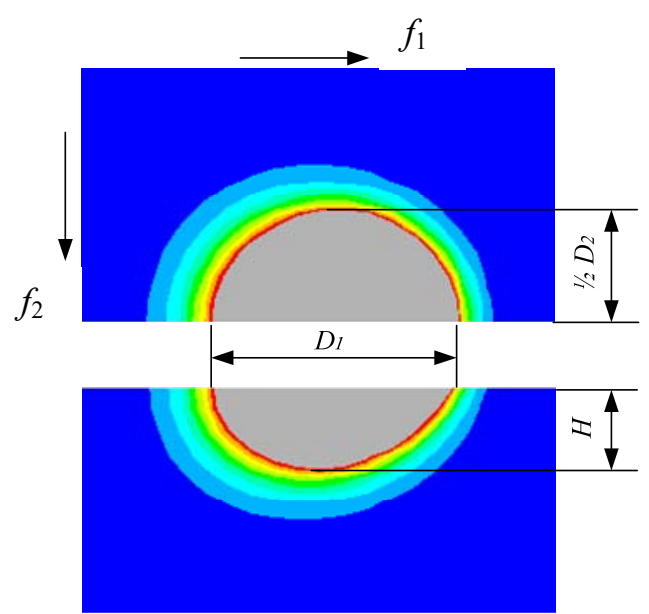

(b) $10.125 \mu \mathrm{J}, H=3.5 \mu \mathrm{m}$ $k_{\mathrm{l}}=1.189, k_{\mathrm{h}}=0.7368$

Fig.2 ANSYS simulation of the single crater

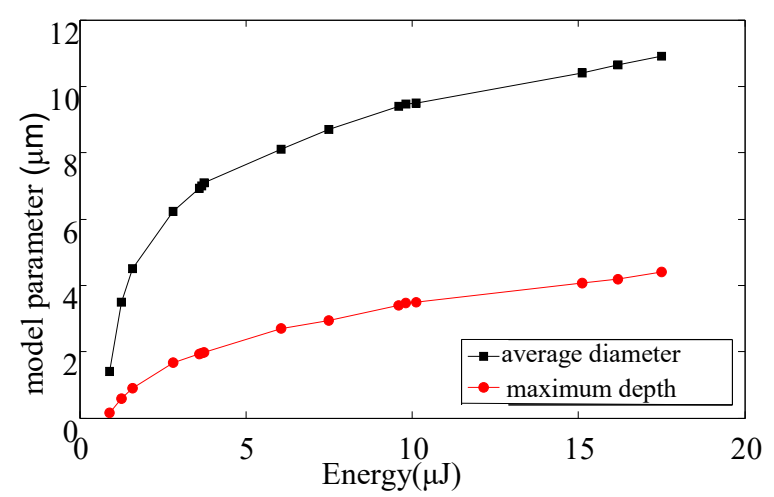

Fig.3 Mathematical model parameter of single crater

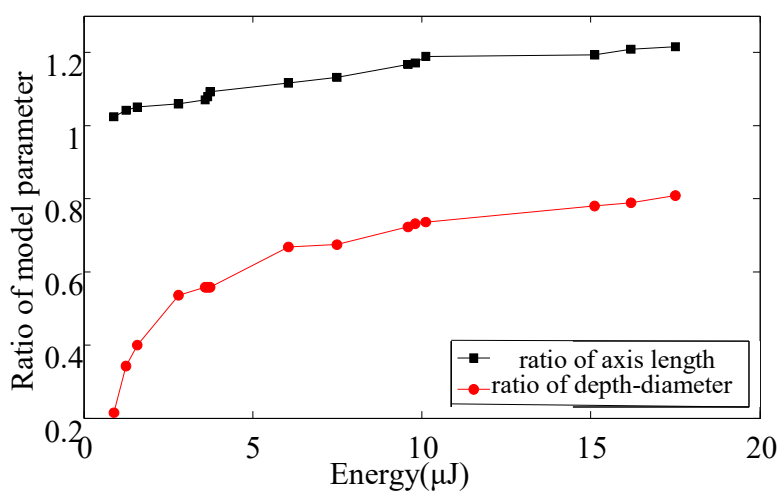

Fig.4 Ratio of parameters of the single crater

\section{Mathematical Model of Multi-crater}

The model of multi-crater overlap. The micro-WEDM surface consists of many approximate spherical peaks and craters which are overlapped by several single craters. According to the micro-WEDM mechanism, discharge craters overlap mainly along two directions including wire electrode vertical movement $f_{1}$ and workbench cross feed $f_{2}$. Fig. 5 shows that there are two craters overlapping the center one in each direction and then form a whole overlap unit. The craters overlap greatly in A, B direction and it can reflect the topography characteristic of micro-WEDM surface.

The real depth of discharge crater he, namely, the absolute value sum of surface peak height and 
valley depth, can be analyzed by overlap unit. The mathematical model of multi-crater is shown in Fig.6.

Overlap Coefficient. The overlap coefficient $\theta$ was introduced to analyze the multi-crater overlap phenomenon. The formula is given as (1):

$$
\left\{\begin{array}{l}
\theta_{1}=\frac{2 l_{1}}{D_{1}} \\
\theta_{2}=\frac{2 l_{2}}{D_{2}}
\end{array}\right.
$$

Here, $l_{1}$ is the crater overlap length along wire electrode movement direction, $l_{2}$ is along cross feed. $D_{1}$ is the crater diameter of long axis and $D_{2}$ is short axis. $\theta_{1}$ is the overlap coefficient along wire electrode movement $\left(0 \leq \theta_{1} \leq 2\right)$, and $\theta_{2}$ is along cross feed $\left(0 \leq \theta_{2} \leq 2\right)$. When $\theta_{1} \rightarrow 2$, means adjacent craters tend to overlap completely and $\theta_{1} \rightarrow 0$ means non-overlap.

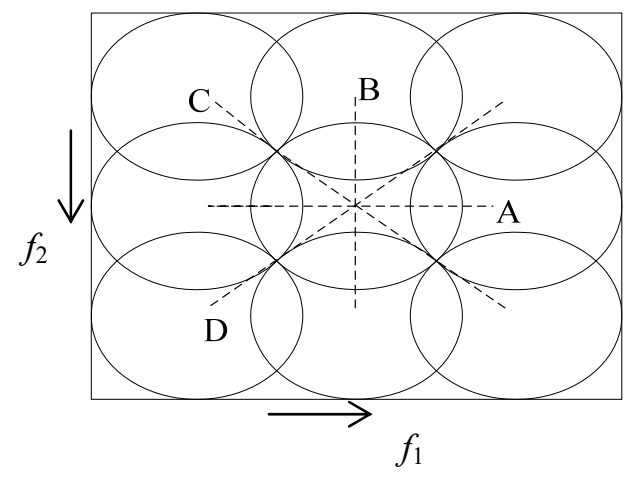

Fig.5 Multi-crater overlap plot

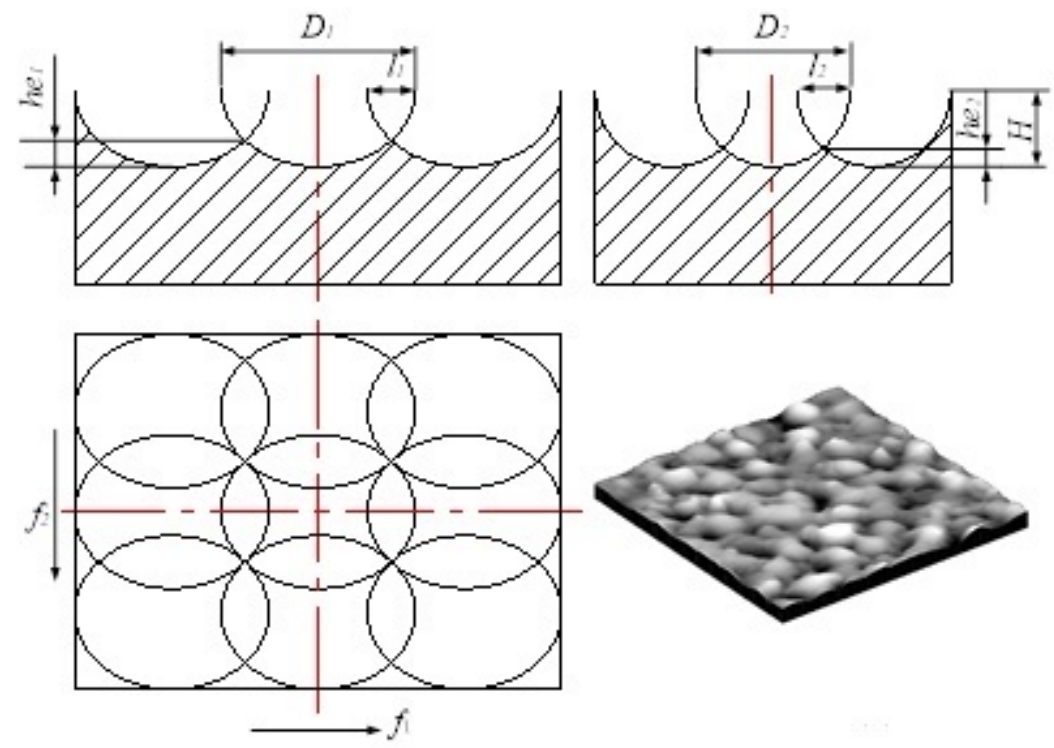

Fig.6 Three-view drawing of multi-crater mathematical model of micro-WEDM surface

\section{Calculation of Three-dimensional Surface Roughness}

The real depth of discharge crater he can be derived through the geometrical relationship of model as follows.

$h e_{1}$, along the wire electrode movement direction, is written as:

$\left\{\begin{array}{lc}h e_{1}=H\left[1-\sqrt{1-\frac{\left(2-\theta_{1}\right)^{2}}{4}}\right] & 0 \leq \theta_{1}<2 \\ h e_{1}=H & \theta_{1}=2\end{array}\right.$

$h e_{2}$, along cross feed, is: 


$$
\left\{\begin{array}{lc}
h e_{2}=H\left[1-\sqrt{1-\frac{\left(2-\theta_{2}\right)^{2}}{4}}\right] & 0 \leq \theta_{2}<2 \\
h e_{2}=H & \theta_{2}=2
\end{array}\right.
$$

The relation between $h e$ and $S a$ (arithmetic average deviation) can be given as

$$
S a=\frac{1}{2 \lambda} \cdot \frac{h e_{1}+h e_{2}}{2}
$$

Where, $\lambda$ is the coefficient of peak and valley. When have more peaks and valleys, $\lambda=1$, otherwise, $\lambda=\sqrt{2}$

The calculation value $S a$ according to formula (4) is compared with the measurement value by SPM, as shown in Fig.7. The two curves almost overlap which means the calculation formula of he and $S a$ is almost correct, and also proves the validity of the multi-crater overlap model.

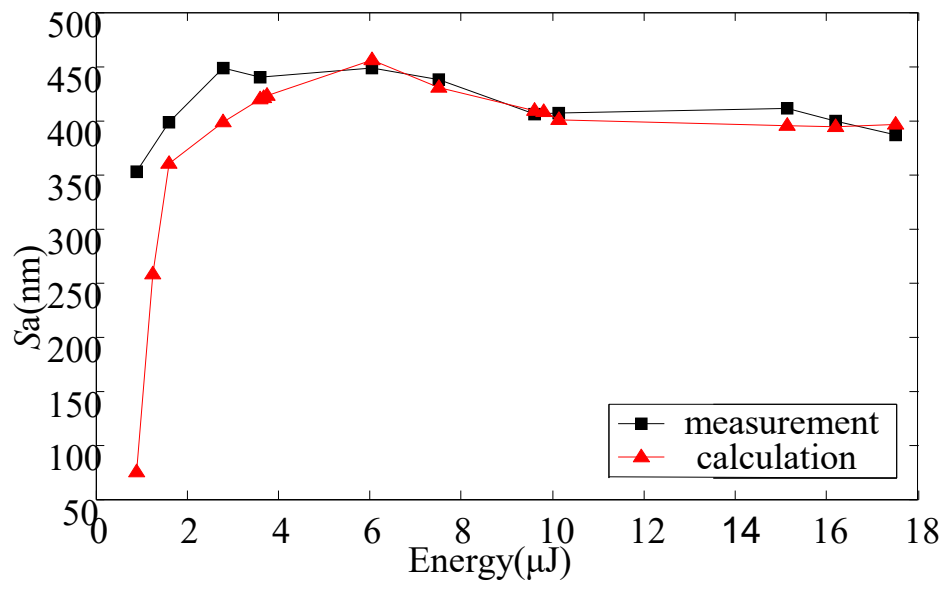

Fig.7 SPM measurement and calculation results of $S a$ with different discharge energy

\section{Conclusion}

The discharge crater of the single pulse of micro-WEDM surface is simulated by using ANSYS software. The mathematical models of single crater and multi-crater are established with overlap theory, which can reflect the geometrical structure and micro-topography characteristic of micro-WEDM surface. The quantitative relationship between the surface micro-structure size he and arithmetic average deviation $S a$ is deduced. Through comparing the numerical simulation result with the real measurement value, this research method is proved to be correct.

\section{Acknowledgement}

This work was financially supported by research fund project of Jiamusi University (Lz2014-002), Heilongjiang Department of Education (12531672) and National Natural Science Foundation of China (51375208).

\section{References}

[1] Sanjeev Kumar, Rupinder Singh, T.P.Singh, et al. Surface modification by electrical discharge machining: A review[J]. Journal of Materials Processing Technology. 2009,209(8):3675-3687

[2] Fuqiang Hu, Hong Zhang, Zhenlong Wang, et al. Surface properties of powder mixed EDM of TaW alloy material. Journal of Nanjing University of Science and Technology. 2008,32(1): 33-36

[3] Zhiwei Yang, Naizhang Yun, Jianning Chen. Basic research of micro-protruding and micro-pit by micro-EDM[J]. Micro-fabrication Technology. 2008, (1):60-65

[4] Segon Heo, Young-Hun Jeong, Byung-Kwon Min, et al. Virtual EDM simulator: 
Three-dimensional geometric simulation of micro-EDM milling processes[J]. International Journal of Machine Tools \& Manufacture. 2009,49(12-13): 1029-1034

[5] Zhidong Liu, Guozhu Cheng, Jianjun Li. Finite element analysis and parameter verification of temperature field in WEDM[J]. China Mechanical Engineering, 2010,21(1):38-41

[6] Guozhu Cheng, Zhidong Liu, Zongjun Tian, et al. Temperature field analysis based on ANSYS in WEDM [J]. Electromachining \& Mould, 2008, (6):24-26 